\title{
A Simple Method for Determining Surface Porosity Based on SEM Images Using OriginPro Software
}

\author{
Mikrajuddin Abdullah and Khairurrijal \\ Physics of Electronic Materials Research Division, \\ Faculty of Mathematics and Natural Sciences, \\ Bandung Institute of Technology, \\ Jalan Ganeca 10 Bandung 40132, Indonesia \\ e-mail:din@fi.itb.ac.id
}

\begin{abstract}
We proposed a simple method for predicting surface porosity of materials based on scanning electron micrograph (SEM) images. The porosity was determined by calculating volume beneath the surface and volume beneath a flat surface, the height of which is equal to maximum height of any point on the sample's surface. The SEM image was firstly converted into two-dimensional matrix of gray scale and all procedures were performed using instructions provided by OriginPro software. The predicted results were in good agreements with direct calculation and measurement of the samples porosities.
\end{abstract}

Keywords: Surface porosity, OriginPro, SEM.

\section{Introduction}

Researches in porous materials are of interest for developing high surface area catalysts, low dielectric constant materials, high quality ceramics, strong composites, filters, etc. In general, pore size and pore distribution determine the performance of many materials.

For bulk materials, the porosity can be determined easily, for example by measuring dimension and weighting mass of the material, by mercury intrusion porosimetry, by Brauner-EmmetTeller (BET) method, etc. The first method can give accurately the porosity of materials containing either connected or unconnected pores. The second and the latter methods might give inaccurate results when used to measure porosity of materials containing unconnected pores. Mercury used in the intrusion method and gas molecules used in the BET method are unable to reach isolated pores inside the materials. In addition, the overall methods, however, become inaccurate when used to detect the porosity of samples, which is very small in size. For example, those methods might be inaccurate for determining the porosity of porous thin film, because the amount of liquid and adsorbed gas might be detected inaccurately. Furthermore, the weighing method is useless because it is difficult to weigh the mass of porous layer that has been deposited on a substrate. For small-sized materials, therefore, alternative method is needed to determine the porosity more accurately.

In literatures, various methods have been introduced to determine the porosity of materials at various sizes. To date, there are backscattering scanning electron microscopy ${ }^{1,2)}$ and synchrotron $\mathrm{X}$ ray micro-computer tomography ${ }^{3)}$. For fine granied materials, authors have employed various methods such as metal injection method $^{4)}$, magnetic susceptibility measurement ${ }^{5)}$, TEM image ${ }^{6)}$, neutron scattering ${ }^{7)}$, and NMR spectroscopy ${ }^{8)}$. For coarse grained materials, confocal microscopy ${ }^{9)}$ and X-ray tomography ${ }^{10)}$ methods have been used by some authors. Some of those methods are very difficult to use or need sophisticated (expensive) equipments as well as need complicated processes or calculations to find the porosity.

Despite the advantageous and disadvantageous of the current methods, in this work, we introduced a simple and might be an alternative approach to predict the porosity of small as well as large sizes materials based on their SEM images. This approach, of course, is unable determine three-dimensional porosity of the material because SEM is unable to observe images of inner parts of the material. Therefore, we called the results obtained in this method as surface porosity. If we cut the material into slices and observe the porosity of each slice's surface, we might extend the method to gather information of three-dimensional porosity by some kinds of interpolation or averaging procedures.

\section{Method}

\subsection{Surface porosity}

Generally, the surface of material is usually not flat, and it is considered to be flat when the roughness scale is negligibly small compared to a certain length criterion. For example, a porous surface is called flat if the roughness scale is negligibly small compared to pore dimension. 


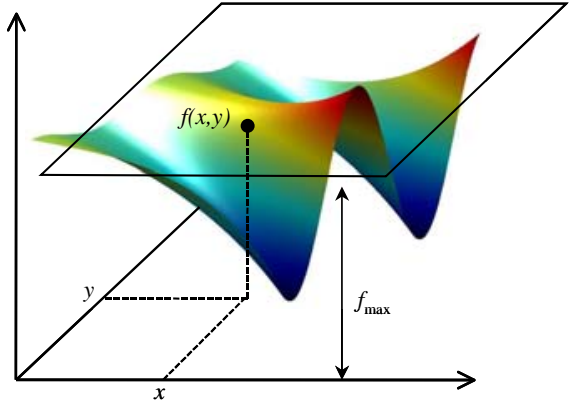

Figure 1. Example of surface of material and its coordinates.

We define surface porosity is this study as following. Consider a surface represented by a function $f(x, y)$ (Fig. 1). This function represents the height of surface at coordinates $x$ and $y$ measured from a certain base plane. The space beneath the surface is solid material while the space above the surface is empty. The volume of solid material beneath the surface is

$$
V_{\text {solid }}=\int_{x_{\min }}^{x_{\max }} \int_{y_{\min }}^{y_{\max }} f(x, y) d x d x
$$

with $x_{\min }, x_{\max }, y_{\min }$, and $y_{\max }$ are boundaries of the surface at coordinates $x$ and $y$ (the boundaries of the surface when projected at the base plane).

Suppose $f_{\max }$ is the maximum height of the surface measured from the base plane at any coordinates $x$ and $y$, where $f(x, y) \leq f_{\max }$ for all $x$ and $y$. We generate a plane parallel to the base plane, where distance from the base plane is $f_{\max }$. We define the total volume of material when pore is absent as volume of space beneath the flat surface of height $f_{\max }$ and bounded by $x_{\min }, x_{\max }, y_{\min }$, and $y_{\max }$. This total volume simply satisfies

$$
V_{\text {total }}=f_{\text {max }}\left(x_{\text {max }}-x_{\min }\right)\left(y_{\text {max }}-y_{\text {min }}\right)
$$

The porosity of the surface is written as

$$
\phi=1-\frac{V_{\text {solid }}}{V_{\text {total }}}
$$

\subsection{Calculation procedures}

To calculate the surface porosity we employed facilities in OriginPro software. At this work we used OriginPro version $8 e$ to calculate the surface porosity based on the SEM images of surfaces. The steps of calculating the porosity are as following.

a) Open worksheet of the OriginPro.

b) Place cursor at a certain cell is the worksheet, click the right button of mouse and select Insert Images from Files.... Follow all instructions until the image file is loaded at the selected cell.

c) Click Image instruction in the OriginPro menu and select Conversion and then click Convert to Data instruction. These instruction steps are used to convert the image into two-dimensional matrix of gray scale. The element of the matrix is gray scale value at a certain location (expressed in pixel). The dimension of the matrix is the same as the pixel dimension of the SEM image. This dimension can be checked using Matrix instruction in the OriginPro menu and click Set Dimensions.

d) The volume of space beneath the sample's surface can be calculated using instruction provided by OriginPro. Select Analysis instruction in the OriginPro menu, then Mathematics and finished by 2D Volume Integrate.... We will then find volume of space beneath the surface in the Log window. This is the $V_{\text {solid }}$ expressed in Eq. (1).

e) The total volume of space beneath the flat surface is obtained by firstly calculating the area of projection of sample's surface on $x-y$ plane. To know dimension of the projected surface, we open Plot instruction in the OriginPro and select any one of 3D Surface instruction. The dimension of the projected surface is indicated by the values in $x$ and $y$ axis.

\section{Results and Discussion}

At first, we need to confirm the correctness of the proposed approach by calculating the porosity of certain surfaces where analytical calculation is available. We compared the results of the analytical calculation and the proposed approach for porosity of a surface formed by self-organized monodipersed particles of known diameter, as shown in Fig. 2. This surface was made by dropping a drop of polystyrene latex colloid on surface of a silicon wafer and then evaporated slowly at temperature of $40{ }^{\circ} \mathrm{C}^{11,12)}$. The colloid was supplied by the Japan Synthetic Rubber company. As can be seen in Fig. 2, the twodimensional organization of particles is perfectly hexagonal in structure.

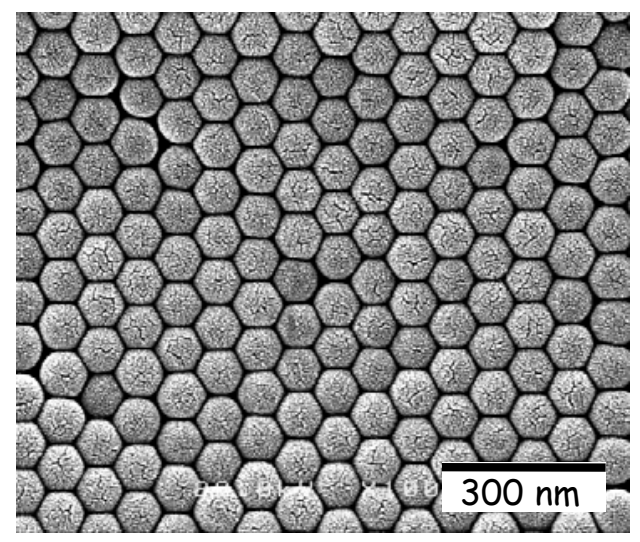

Figure 2. Self-organized polystyrene latex beads on a silicon waver.

To calculate the surface porosity analytically, look at Fig. 3. We selected the base plane as a plane dividing a particle into two parts (upper and bottom hemisphere). The distance of this base plane to top of 
the particles is equal to particle radius, $R$. The porosity of surface was determined by merely calculating the space beneath plane triangle $\mathrm{OAB}$ and the space beneath curved surface of the particle, the sides of which are bounded by triangle OAB. The volume of space beneath the triangle $\mathrm{OAB}$ is $R^{3} \tan 30^{\circ}$. The volume of space beneath the particle surface bounded by triangle is $(1 / 6)(1 / 2)(4 \pi / 3) R^{3}=\pi R^{3} / 9$. The porosity of the surface becomes $\phi=1-\left(\pi R^{3} / 9\right) /\left(R^{3} \tan 30^{\circ}\right)=\pi \sqrt{3} / 9=0.6$. Using the computation procedures explained in 2.2 we obtained the porosity of 0.59 , which is very close to the analytical result.
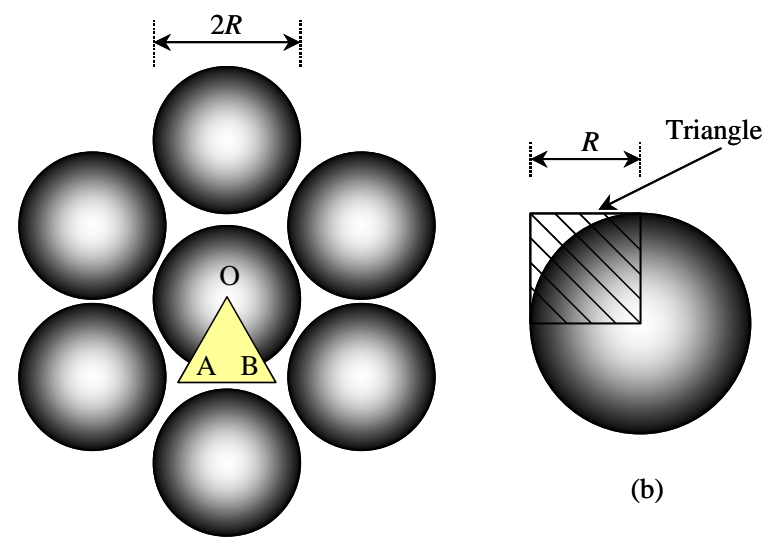

(b)

(a)

Figure 3. (a) Geometrical scheme of self-organized polystyrene beads on silicon wafer and (b) elements of volume for calculating the porosity.

As another proof for our approach, we determined the porosity of titania pellet made by pressing tinania powder into cylindrical shaped and then sintered some minutes at $800{ }^{\circ} \mathrm{C}^{13,14)}$. The titania particles were anatase in structure and purchased from Bratachem (Indonesia). The SEM images of the sample taken at different magnifications $(40,000 \times)$ and $(20,000 \times)$ are shown in Fig. 4. Using procedures explained in 2.2 we obtained the calculated porosities from images of two magnifications are exactly the same, i.e., 0.69.

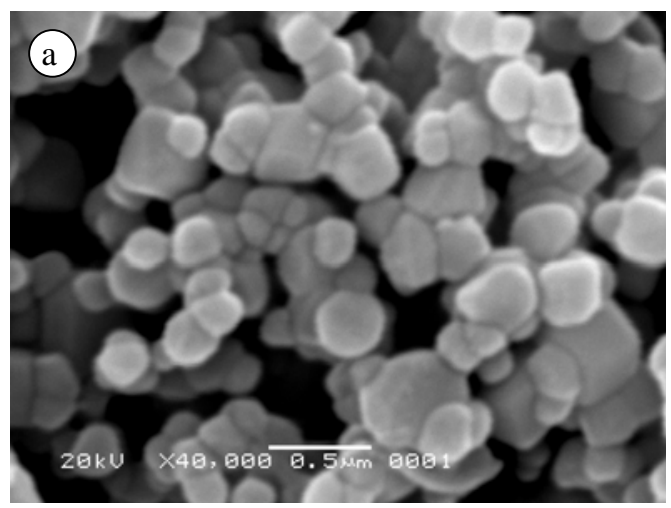

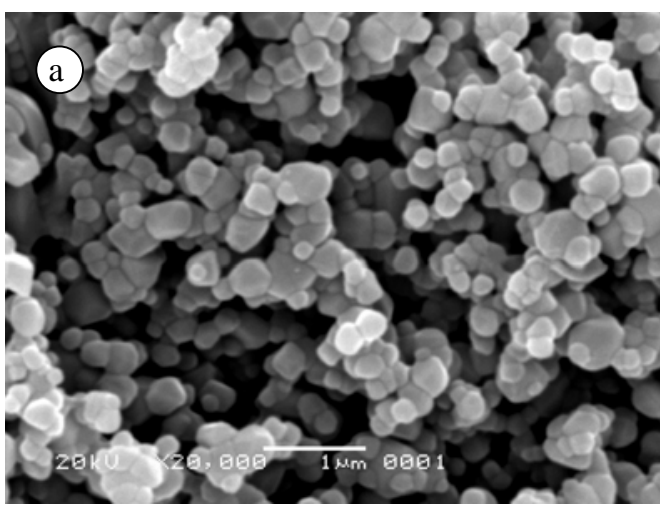

Figure 4. SEM images of titania pellet made by pressing tinania powder into cylindrical shaped and then sintered some minutes at $800{ }^{\circ} \mathrm{C}$. (a) Viewed by $40,000 \times$ magnification and (b) viewed by $20,000 \times$ magnification.

We compared this result by measuring the porosity directly. The dimension and mass of the pellet were measured directly. The porosity of the pellet was determined by a simple formula

$$
\phi=1-\frac{M_{\text {meas }}}{\rho V_{\text {meas }}}
$$

with $M_{\text {meas }}, V_{\text {meas }}$, and $\rho$ are, respectively, the measured mass of the pellet, the measured total volume of the pellet, and the mass density of the titania. By using data of titania mass density from references, we obtained the porosity of about 0.71 . This result also confirmed our approach finely.

For both organized polystyrene latex beads and titania pellet samples, the bulk porosity should be equal to the surface porosity because they were made by compacting/arranging particles. Different results of surface and bulk porosities might be obtained in materials where pores have been created by some kinds of natural process such as found in porous stones. The pores in those materials might distribute inhomogeneously.

\section{Conclusion}

The proposed method was successful to predict the surface porosity of materials based on their SEM images using instructions provided by OriginPro. This method is very simple and the result can be obtained quickly. The porosity was determined based on the calculation of volume beneath the surface and the volume beneath a flat surface, the height of which is equal to the maximum height of any point on the sample's surface. The method results were in good agreement with direct calculation and measurement.

\section{References}

1. C.D. Tsakiroglou, et al., A new Approach for the Characterization of the Pore Structure of Dual Porosity Rocks, Chem. Eng. Sci. 64, 847, 2009. 
2. Y. Qin et al., Correlation between SEM measured microstructure and NMR predicted bone porosity 2003 Summer Bioengineering Conference (Key Bicayne, Florida, 25-29 Jun. 2003) p. 1097, 2003.

3. T. Link et al., Synchrotron Tomography of Porosity in Single-Crystal Nickel-Base Alloys, Mater. Sci. Eng. B. 425, 47, 2006.

4. A. Hildebrand and J. L. Urai, Investigation of the Morphology of Pore Space in Mudstones- the first Results, Marine Petrol. Geol. 20, 1185-1200, 2003.

5. L. Esteban, Y. Geraud and J. L. Bouchez, Pore Network Geometry in low Permeability Argillites from Magnetic Fabric Data and Oriented Mercury Injection, Geophys. Res. Lett., 33, L18311, 2006.

6. R. Ziel, A. Haus and A. Tulke, Quantification of the Pore Size Distribution (Porosity Profiles) in Microfiltration Membranes by SEM, TEM, and Computer Image Analysis, J. Membrane Sci. 323, 241, 2008.

7. K.D. Knudsen et al., Pore Characteristics and Water Absorption in a Synthetic Clay, J. Appl. Crystall. 36, 587, 2003.

8. P. Sozzani et al., Nanoporosity of an Organo-clay Shown by Hyperpolarized Xenon and 2-D NMR Spectroscopy, Chem. Commun. 1921-1923, 2006.
9. J.T. Fredrich, B. Menendez and T.F. Wong, Imaging the Pore Structure of Geometarials, Science 268, 276-279, 1995.

10. S. Zabler et al., High Resolution Tomography of Cracks, Voids and Microstructures in Greywacke and Limestone, J. Struct. Geol. 30, 876-887, 2008.

11. Mikrajuddin, F. Iskandar and K. Okuyama, Single Route for Producing Organized Metallic Domes, Dots, and Pores by Colloidal Templating and Over-sputtering, Adv. Mater. 14, 930-933, 2002.

12. Mikrajuddin, F. Iskandar and A. Khairuddin, Two Dimensional Ordered Metallic Particles with Controllable Size and Distance Produced using Simple ion Sputtering, Integral 8, 19, 2003.

13. A. Pritama et al., Fabrication of Microporous Water Filter using Titanium Dioxide Particles, Silica Particles, and Polyethylene Glycol, ITB J. Sci. (accepted), 2009.

14. A. Pritama et al., Titanium Dioxide-based Reusable Microporous Water Filter using Silicon Dioxide as Filler, J Nano Saintek. Special Ed. Aug. 2009 p. 79, 2009. 